\title{
Cognitive behaviour therapy for whom?
}

\section{Stirling Moorey}

In many ways cognitive and behavioural therapies represent the acceptable face of psychotherapy for the general psychiatrist. They are brief, focused, problem-oriented treatments, which take symptoms seriously. They show an affinity for the medical model in their acceptance of diagnostic categories and their commitment to effective evaluation of treatments through randomised controlled trials. The wide applicability of these therapies is also attractive to the general psychiatrist. Cognitive and behavioural techniques are of major importance in the treatment of anxiety disorders, depression, eating disorders, and sexual dysfunctions, and beyond this core group the methods can be applied to enhance coping and change unwanted behaviours in conditions as diverse as cancer, chronic pain, substance abuse, anger control, schizophrenia, and challenging behaviours in people with learning disabilities.

Despite these attractions, relatively few psychiatrists are trained in cognitive behavioural techniques, and there is uncertainty about who should be referred. This article describes the conditions which cognitive behaviour therapy (CBT) can treat, and the characteristics of the patients who are most likely to benefit. It also suggests some ways in which the process of referral can itself be used to increase the chances that the patient will engage in therapy.

\section{Cognitive and behavioural psychotherapies}

Behaviour therapy has its roots in learning theory: classical (Pavlovian) conditioning or operant (Skinnerian) conditioning. The behaviourist is primarily interested in observable behaviour and the way in which environmental events increase or decrease behavioural responses. In its most radical form behaviourism sees internal mental processes, such as thoughts and beliefs, as epiphenomena. Even now some behaviour therapists still consider that behaviour change always precedes cognitive change, and so cognitive techniques can add nothing to behavioural interventions.

By contrast, cognitive therapy (based on the work of pioneers like Beck (1976) and Ellis (1962)) asserts that understanding thoughts, images and beliefs is the most important component of therapy. A cognitive therapist would consider behavioural techniques to work by producing cognitive change. For instance, in the treatment of a phobia the behaviour therapist believes that exposure has a direct effect on the conditioned fear responses, independent of cognitions. The cognitive therapist, however, sees exposure in humans as a form of behavioural experiment which demonstrates that a feared consequence does not occur: change comes from disproving the patient's erroneous belief. The impact of the 'cognitive revolution' on behaviour therapy has been profound, and in practice most behaviour therapists use cognitive techniques alongside conventional behavioural ones, hence the term cognitive behaviour therapy.

Cognitive and behavioural therapies are characterised by a focus on the patient's current life problems. The therapist is active and collaborates with the patient in identifying problems and finding solutions; the problems are defined in a way that allows their severity and frequency of occurrence to be measured to assess change. There is an emphasis on participation in the therapy through the use of self-help assignments between sessions. Within this framework the therapist uses a variety of techniques to produce change. Selfmonitoring of thoughts, beliefs, emotions and

Stirling Moorey is Consultant Psychiatrist with special responsibility for cognitive psychotherapy at the City and Hackney Community Services Trust, and Honorary senior lecturer at the Medical College of St Bartholomew's Hospital. He is one of the co-founders of the Institute of Psychiatry Cognitive Behaviour Therapy Course. His main area of interest is cognitive therapy for patients with cancer. 
behaviours can give information about the problem and also help the patient to begin to get some control over it.

Behavioural techniques include graded exposure to feared situations (phobias/obsessive-compulsive disorder); graded tasks and activity scheduling (depression); removing the reinforcing consequences of maladaptive behaviour; and rewarding adaptive behaviour (challenging behaviours).

Cognitive techniques include daily diaries to identify dysfunctional thoughts and beliefs; labelling types of distortion in negative thoughts; reality testing by questioning the evidence for negative interpretations; setting up experiments to test erroneous beliefs; and cognitive rehearsal of coping with difficult situations.

Length of treatment varies depending on the problem treated. For a simple phobia a few hours of therapist time with self-exposure between sessions is sufficient to produce complete cure, cognitive therapy for depression is traditionally one hour of therapy once a week for 10-20 sessions, while cognitive therapy for personality disorders may require a year of therapy.

\section{Applications of cognitive behaviour therapy}

\section{Exposure for phobias and obsessive-compulsive disorder}

Graded exposure is a simple and effective treatment for specific anxiety disorders. The patient and therapist construct a hierarchy of feared situations, and the patient is encouraged to confront them in a graded way. Exposure is more effective if it is in real life rather than imagination (Emmelkamp \& Wessels, 1975), prolonged rather than brief (Stern \& Marks, 1973) and practised regularly with selfexposure tasks (McDonald et al, 1978). Exposure is most effective with simple phobias such as animal phobias, where the phobic stimulus is clear. About two-thirds of agoraphobics are successfully treated with exposure alone, and these gains are maintained at 2 year follow-up (Munby \& Johnston, 1980). Social phobia has also been effectively treated with exposure (Butler et al, 1984). Despite these successes a significant percentage of patients do not improve with exposure alone, and adding anxiety management or cognitive procedures may enhance the effects of exposure (Butler et al, 1984).

Exposure has also proved successful in the treatment of obsessive-compulsive disorder
(OCD). Obsessional patients try to neutralise fears by rituals, such as checking, handwashing etc. Therapy consists of graded and prolonged exposure to the feared stimuli (e.g. contamination) with the additional component of preventing any neutralising rituals (response prevention). A combination of exposure and response prevention seems to be most effective, producing a median success rate of $75 \%$ improvement in patients who complete treatment. However, because of treatment refusal, dropout or failure, only $50 \%$ of all patients with OCD are actually helped (Salkovskis 1989).

Obsessional ruminations without overt rituals are more difficult to treat. Exposing the patient to the ruminations by using a loop tape played on a personal stereo is sometimes effective. There is evidence for the efficacy of serotonin re-uptake inhibitors in treating OCD, but the long-term outcome has not been satisfactorily evaluated: there is a high relapse rate when drug therapy is discontinued. With behaviour therapy, on the other hand, gains in phobics (Fava et al, 1995) and obsessionals can be maintained at 4 year followup.

Exposure with or without the addition of cognitive restructuring should therefore be considered the treatment of choice for phobias and OCD. In uncomplicated cases a referral for CBT should be made directly. Patients with OCD who are clinically depressed, whose obsessional symptoms are so severe they could not immediately use therapy, or who are unwilling to take part in treatment, should be treated with clomipramine or a SSRI; but one of the main aims of treatment should be to prepare the patient for a course of CBT.

\section{Generalised anxiety, panic disorder and hypochondriasis}

These conditions differ from phobias and OCD in that there is not a readily identifiable external stimulus to which the patient can be exposed. Behaviour therapy attempts to identify internal stimuli such as physiological cues for panic, and to expose the patient to them. The cognitive model of anxiety stresses the overestimation of the risk of catastrophe, misperception of danger and underestimation of coping abilities. Cognitive therapy challenges unrealistic thoughts and sets up experiments to test the negative predictions associated with anxiety.

The cognitive model of panic stresses the specific interpretations the patient makes about the physical symptoms of anxiety. According to this model, catastrophic misattributions are made 
about symptoms such as palpitations, dizziness, faintness and breathlessness, which are really due to autonomic manifestations of anxiety, but are seen as signs of impending death, madness or loss of control. A vicious circle develops in which the automatic thoughts produce more anxiety and therefore more symptoms. Therapy consists of helping the patient to test their attributions through logical discussion and setting up behavioural experiments. Clarket al (1994) compared cognitive therapy, imipramine, applied relaxation and a waiting list control. The active treatments were all more effective than the waiting list, and cognitive therapy was the most effective treatment after completion and at follow-up.

The cognitive approach to hypochondriasis is similar to panic: patients misinterpret bodily symptoms as signs of physical illness, but do not believe they are about to die immediately. Reassurance seeking and avoidance act to maintain the hypochondriacal beliefs. Cognitive therapy develops an alternative explanation for the patient's symptoms, while behaviour therapy stresses overcoming avoidance and a ban on reassurance seeking. Preliminary studies show that both treatments may be helpful for these difficult patients (Salkovskis \& Warwick, 1986). Whereas brief CBT can be effective in hypochondriacal conditions which have strong obsessional and anxious features, with other forms of somatisation (e.g. somatisation disorder) interpersonal and personality factors may predominate making it difficult to engage in collaborative problem-

Box 1 Which patients benefit from cognitive and behavioural therapies?

Psychological treatment of choice Simple phobia

Agoraphobia

Social phobia

Obsessive-compulsive disorder

Generalised anxiety disorder

Panic disorder

Depression

Bulimia

Sexual dysfunction

Evidence of efficacy

Post-traumatic stress disorder

Substance misuse

Sexual deviancy

Anorexia

Relapse prevention in schizophrenia

CBT still experimental

Personality disorder

Managing delusions and hallucinations solving. These patients may require longer term CBT. Patients with somatic conditions are being increasingly treated with CBT, and there are promising preliminary results with chronic fatigue syndrome.

Many patients with anxiety disorders will respond to a combination of education about their symptoms, support and drug therapy. Patients experiencing problems with long-term prescribing of benzodiazepines often want to learn anxiety management strategies which they can apply themselves. Therefore, if symptoms do not resolve within a few weeks a CBT referral should be considered.

\section{Cognitive therapy for depression}

Beck's cognitive model of depression states that the depressed person has a negative view of the self, the world and the future (Beck et al, 1979), which leads to the distortion of information to fit this negative set. Cognitive therapy is now widely accepted as an effective treatment for nonpsychotic, unipolar, out-patient depressives. Behavioural techniques such as graded task assignment and activity scheduling are used to distract the patient from negative thoughts and provide experiences of success and pleasure. Patients are then taught to identify and challenge their distorted thinking. Finally the dysfunctional assumptions which underlie the depressive thinking and make the patient vulnerable to depression are challenged. In numerous studies cognitive therapy has proved as effective, or more effective than tricyclic antidepressants (see Dobson, 1989 for a meta-analysis of 28 studies). One large multi-centre trial found that cognitive therapy did less well than tricyclic antidepressants and interpersonal therapy with more severely depressed patients (Elkin et al, 1989). Cognitive therapy claims to prevent relapse of depression and several follow-up studies have supported this claim (Blackburn et al, 1986).

Most depressed patients will respond well to antidepressant medication, and it is also not practical for all to receive cognitive therapy. A number of factors might be considered when deciding which depressed patient should be referred. Firstly, not all patients are able or willing to take antidepressants: some refuse medication, others cannot tolerate side-effects, and a small number have medical conditions which preclude drug treatment. Cognitive therapy may be the best first line of treatment in these cases. Secondly, not all patients make a complete recovery with pharmacotherapy. This sort of chronic low grade 
depression can be very disabling, and may respond to cognitive therapy. Finally, there is now strong evidence that depression is a relapsing condition. Maintenance antidepressants prevent relapse, but their protective effect is lost when treatment is ended. Because of its possible effects on relapse prevention, cognitive therapy should be considered in any patients with recurrent depressive illness.

\section{Eating disorders}

Fairburn et al (1986) suggest that bulimics have a tendency to judge self-worth in terms of shape and weight. Strict dietary control and perfectionistic attitudes mean that any lapse from the diet is seen as catastrophic and leads to a temporary abandonment of all control over eating. Therapy consists of monitoring binges and food intake, adopting a more normal dietary pattern and challenging maladaptive beliefs. Fairburnet al (1991) compared cognitive behaviour therapy, behaviour therapy and interpersonal therapy. All treatments produced improvements, but cognitive therapy was more effective than the other treatments in modifying disturbed attitudes to shape and weight and reducing extreme dieting. Cognitive behaviour therapy has been found to be more effective than imipramine (Mitchell et al, 1990). CBT for anorexia nervosa is less well established. This evidence suggests that CBT should be the primary intervention in patients with bulimia nervosa. The acute stage of anorexia nervosa often requires hospital treatment to induce weight gain. There may be a place for CBT in challenging maladaptive attitudes about food, shape and weight once the patient is out of the life threatening stage of the illness.

\section{Other conditions}

In general adult psychiatry, promising results are being obtained with patients with borderline (Linehan, 1993) and other personality disorders (Becket al, 1991). Behavioural techniques have had a place in the rehabilitation of patients with longterm mental illness for many years. Recent developments in work with schizophrenia have used cognitive behavioural family interventions to teach problem solving skills (Falloon, 1988), reduce high expressed emotion (Leff $e t a l, 1982$ ) and to teach patients and families to recognise early signs of relapse. Another exciting development is the application of cognitive behavioural principles to coping with psychotic symptoms and even challenging delusional ideas.

Box 1 lists the conditions for which CBT should be considered the psychological therapy of choice.

\section{Factors influencing outcome in CBT}

\section{Coexisting diagnoses}

Because both cognitive and behavioural techniques require the commitment and active participation of the patient, coexisting diagnoses such as depression and substance misuse can interfere with treatment. Secondary depression and anxiety, and also poor work, social or marital adjustment is associated with a poorer response to behaviour therapy for phobias and OCD (Marks, 1987). These conditions respond to exposure if clinical depression is first treated with drugs (Baer et al, 1985).

Social phobics and to a lesser extent agoraphobics can resort to alcohol and drugs as a way of dampening down anxiety. Alcohol misuse and excessive use of benzodiazepines can interfere with the effectiveness of exposure, and it may be necessary to treat these conditions before cognitive behaviour therapy is commenced.

\section{Chronicity and severity}

In general the more severe and chronic a disorder the poorer will be the outcome with any form of therapy. Marks (1987) claims that for phobias and OCD, chronicity does not augur poorer outcome with exposure.

In out-patient cognitive therapy for depression, chronicity and severity are associated with a poor outcome. Modification of standard CBT may be needed when treating severely depressed inpatients. The finding that chronicity and severity predict poor outcome is not specific to cognitive therapy: these variables also predict poor response to antidepressants. Some studies have found a combination of cognitive therapy and antidepressants to be superior to either alone, while others have not found any advantage of combined treatment. In clinical practice it is rare for a patient with moderate to severe depression to be referred for cognitive therapy without also receiving drug therapy. The NIMH (Elkin et al, 1989) study suggests that cognitive therapy alone may not be the most effective treatment for severe depression.

\section{Psychosocial factors}

The association of depression with psychosocial factors might suggest that CBT will be less effective in socially disadvantaged groups and in people facing major life stress. In fact, both CBT and tricyclic antidepressants seem to be equally 
effective in patients experiencing high social stressors (Garvey et al, 1994).

Personality and interpersonal relationships

There is some evidence that the presence of a comorbid personality disorder predicts poor outcome in generalised anxiety (Noyes et al, 1990), agoraphobia (Fava et al, 1995) and bulimia nervosa (Rossiter et al, 1993). Sanderson et al (1994) found that personality disordered patients with GAD did not do less well but were more likely to drop out.

Interpersonal and relationship difficulties are often felt to be clinically important in agoraphobia: quality of marriage does not predict short-term outcome (Cobb et al, 1984) but may be associated with long-term follow-up. A high proportion of agoraphobic women report marital difficulties, but this is not a contraindication to behaviour therapy, since marital satisfaction improves following successful treatment with exposure .

Personality disorder should not be considered an absolute contraindication to referral, but treatment may take longer than with more straightforward patients (Beck et al, 1991).

\section{Cognitive factors}

Patients are often referred for cognitive behaviour therapy because they display conspicuously negative thinking patterns, with the assumption that they are therefore ideal candidates. In fact, cognitive variables such as hopelessness, frequency of negative thoughts, cognitive distortions and maladaptive assumptions are highly correlated with mood and are not predictive of response to therapy in their own right. Patients who do better with cognitive therapy actually have lower scores on dysfunctional attitudes (see Moorey, 1989 for a review of patient factors associated with outcome in CBT for depression). Patients with more cognitive distortions often have life-long, fixed negative beliefs about themselves which are not amenable to standard cognitive interventions, but may need longer, schema-focused therapy (Beck et al, 1991).

These findings would suggest that there are not any clear cognitive predictors of outcome with CBT, and that the degree of negative thinking should not be a factor in deciding whether or not to make a referral.

It is also sometimes assumed that patients need to be of a certain intellectual standard to engage in CBT or that they need to have a high degree of education. In fact, education and IQ have no effect on the outcome of cognitive therapy for depression.
Box 2 Making a referral

(i) Is CBT a proven treatment for this diagnostic group?

(ii) Are there any contraindications? e.g. severe depression, substance misuse

(iii) Is the patient motivated to work psychologically?

(iv) Can the patient identify problems and goals to work on?

(v) Does the patient accept the therapeutic model?

(vi) Is the patient prepared to carry out selfhelp assignments?

\section{Motivation for change}

Clinically the patient's desire to find relief from symptoms, and to solve personal problems is considered important in assessing suitability for therapy. From a research perspective, attempts to predict outcome by measuring a unitary variable of 'desire to change' have not been successful. Even if it seems obvious that a symptom is disabling, it does not mean that the patient wishes to deal with it. The therapists' time can be saved if the referrer checks that the patient actually does want to give up the particular symptom.

\section{Goals for therapy}

In general, $\mathrm{CBT}$ is more applicable if patients have clear ideas of the problems they wish to work on and clear goals for therapy. "Understanding myself better" does not augur well as a goal, whereas "being able to drive more than 10 miles on my own without anxiety" does. CBT is a problem solving therapy and works best when patients have problems in the here and now. Patients who wish to gain insight, to explore their past or resolve conflicts are more suitable for dynamic psychotherapy. Again it is helpful for the clinician to discuss this explicitly with the patient before referring for therapy.

\section{Acceptance of the therapeutic model}

Frank (1973) has argued that therapy is most effective if the patient feels confident in the therapist and accepts the therapist's explanation of the cause and cure of their problems. In CBT this applies not only to what goes on within the session, but also to the patient's compliance with self-help assignments outside the session. There is some evidence that patients who already think in a problem-solving way are more likely to respond 
Box 3 Self-help material for patients

Exposure for phobias and obsessive-compulsive disorder

Living with Fear. By Isaac Marks. McGraw Hill

Cognitive therapy

Cognitive Therapy and the Emotional

Disorders. By Aaron T. Beck. Penguin.

Cognitive therapy for depression

Coping with Depression. By Ivy Blackburn. Chambers.

The Feeling Good Handbook. By David

Burns. Morrow.

CBT for eating disorders

Getting Better Bit(e) by Bit(e). By Ulrike

Schmidt and Janet Treasure. Sage.

to CBT. Simons et al (1985) found that a measure of 'learned resourcefulness' (a tendency to use problem-solving and self-control skills) predicted good response to cognitive therapy and poor response to drug treatment. While some studies have replicated this finding, others have not. Fennel \& Teasdale (1987) found that patients who had a positive response to a booklet describing cognitive therapy and went on to have a successful first homework assignment recovered more rapidly. The effectiveness of exposure for phobias and obsessive -compulsive disorder depends to a large extent on the patients willingness to engage in self-directed exposure homework (Marks, 1987). In cognitive therapy for depression homework compliance is associated with better outcome. Burns \& HolenHoeksema (1991) found that expectations about the value of coping strategies did not correlate with outcome, but willingness to carry out self-help assignments and homework compliance did.

Taken together these findings suggest that individuals who tend to think in a problem-focused way may find it easier to accept the rationale for cognitive behaviour therapy and so do better in therapy. Both the behavioural and more cognitive forms of CBT work better if the patient is prepared to engage in self-help assignments. An important part of assessment for CBT must therefore involve assessing the patients' acceptance of the therapeutic model. It is worth describing the rationale for therapy (principles of exposure for phobias and obsessions, and the cognitive model for depression, anxiety, eating disorders) to the patient before a referral is made, while emphasising the importance of homework assignments. If a patient resolutely refuses to consider that the psychological model has merit, or indicates that they will not be able to do homework, a referral is probably not worth- while. Some general psychiatrists take this one stage further and give the patient reading material about CBT. This is in effect a behavioural experiment. If the patient responds negatively to the information in the literature, or does not even carry out the assignment this may be a contraindication.

\section{Referral considerations}

The increasing evidence for the wide range of applications of CBT in psychiatry means that many of the patients a psychiatrist encounters in outpatients could potentially benefit from this approach. Availability of CBT is still limited. There are increasing numbers of trained nurse behaviour therapists but they are not all trained in cognitive therapy. Most clinical psychology departments will provide CBT, but in psychiatry there are still only a handful of consultant psychotherapy posts which are cognitive behavioural rather than psychoanalytic. Given the need to be selective in which patients are referred, these guidelines may be helpful in choosing the patients most likely to benefit from CBT. But in the long run they are no substitute for providing adequate resources, and training psychiatrists themselves in the application of cognitive behaviour therapy.

\section{Acknowledgement}

I am grateful to Mrs Ruth Williams who made helpful comments on an earlier draft of this paper.

\section{References}

Baer, B. L., Minichiello, W. E. \& Jenike, M. A. (1985) Behavioural treatment in two cases of OCD with concomitant bipolar affective disorder. American Joumal of Psychiatry, 142, 358-360.

Beck, A. T. (1976) Cognitive Therapy and the Emotional Disorders. New York: International Universities Press.

-, Rush, A. J., Shaw, B. F., et al (1979) Cognitive Therapy of Depression. New York: Guilford Press.

-, Freeman, A., et al (1991) Cognitive Therapy of Personality Disorders. New York: Guilford Press.

-, Eunson, K. M. \& Bishop, S. (1986) A two-year naturalistic follow-up of depressed patients treated with cognitive therapy, pharmacotherapy and a combination of both. Journal of Affective Disorders, 10, 67-75.

Burns, D. D. \& Holen-Hoeksema, S. (1991) Coping styles, homework compliance, and the effectiveness of cognitivebehavioral therapy. Journal of Consulting and Clinical Psychology, 59, 305-311.

Butler, G., Cullington, A., Munby, M., et al (1984) Exposure and anxiety management in the treatment of social phobia. Journal of Consulting and Clinical Psychology, 52, 642-650.

Clark, D. M., Salkovskis, P. M., Hackmann, A., et al (1994) A comparison of cognitive therapy, applied relaxation and 
imipramine in the treatment of panic disorder.British Journal of Psychiatry, 164, 759-769.

Cobb, J. P., Mathews, A. M., Childs-Clark, A., et al (1984) The spouse as co-therapist in the treatment of agoraphobia. British Journal of Psychiatry, 144, 282-287.

Dobson, K. S. (1989) A meta-analysis of the efficacy of cognitive therapy for depression. Journal of Consulting and Clinical Psychology, 57, 414-419.

Elkin, I., Shea, T., Watkins, J. T., et al (1989) NIMH Treatment of depression collaborative research program. I. General effectiveness of treatments. Archives of General Psychiatry, 46, 971-982.

Ellis, A. (1962) Reason and Emotion in Psychotherapy. New York: Lyle Stuart.

Emmelkamp, P. M. G. \& Wessels, H. (1975) Flooding in imagination versus flooding in vivo for agoraphobics. Behaviour Research and Therapy, 13, 7-15.

Fairburn, C. G., Cooper, Z. \& Cooper, P. J. (1986) The clinical features and maintenance of anorexia nervosa. In Handbook of Eating Disorders: Physiology, Psychology and Treatment of Obesity, Anorexia and Bulimia, (eds K. D. Brownell \& J. P. Foreyt). New York: Basic Books.

Fairburn, C. G., Jones, R., Peveler, R. C., et al (1991) Three psychological treatments for bulimia nervosa. Archives of General Psychiatry, 48, 453-469.

Falloon, I. R. H. (1988) Handbook of Behavioural Family Therapy. London: Unwin Hyman.

Fava, G. A., Zielezny, M., Savron, G., et al (1995) Long-term effects for behavioural treatment of panic disorder with agoraphobia. British Journal of Psychiatry, 166, 87-92.

Fennell, M. J. V. \& Teasdale, J. D. (1987) Cognitive therapy for depression: individual differences and the process of change. Cognitive Therapy and Research, 11, 253-271.

Frank, J. (1973) Persuasion and Healing: A Comparative Study of Psychotherapy (2nd edn). Baltimore, MD: Johns Hopkins University Press.

Garvey, M. J., Hollon, S. D. \& DeRubeis, R. J. (1994) Do depressed patients with higher pre-treatment stress levels respond better to cognitive therapy than imipramine? Journal of Affective Disorders, 32, 45-50.

Leff, J., Kuipers, L., Berkowitz, R., et al (1982) A controlled trial of intervention in the families of schizophrenic patients. British Journal of Psychiatry, 141, 121-134.

Linehan, M. M. (1993) Cognitive-Behavioral Treatment of Borderline Personality Disorder. New York: The Guilford Press.

McDonald, R., Sartory, G., Grey, S. J., et al (1978) Effects of selfexposure instructions on agoraphobic out-patients. Behaviour Research and Therapy, 17, 83-85.

Marks, I. M. (1987) Fears, Phobias and Rituals: Panic, Anxiety, and their Disorders. New York: Oxford University Press.

Mitchell, J. E., Pyle, R. L., Eckert, E. D., et al (1990) A comparison study of antidepressants and structured group therapy in the treatment of bulimia nervosa. Archives of General Psychiatry, 47, 149-157.

Moorey, S. (1989) Cognitive therapy of depression: patient factors related to outcome. In Cognitive Psychotherapy: Stasis and Change (eds W. Dryden \& P. Trower). London: Cassell.

Munby, M. \& Johnston, G. W. (1980) Agoraphobia: the longterm follow-up of behavioural treatment. British Journal of Psychiatry, 137, 418-427.

Noyes, R. J., Reich, J., Christiansen, J., et al (1990) Outcome of panic disorder. Archives of General Psychiatry, 47, 809-818.

Rossiter, E. M., Agras, W. S., Telch, C. F., et al (1993) Cluster B personality disorder characteristics predict outcome in the treatment of bulimia nervosa. International Journal of Eating Disorders, 13, 349-357.

Salkovskis, P. M. (1989) Somatic disorders. In Cognitive Behaviour Therapy for Psychiatric Problems: A Practical Guide. (eds K. Hawton, P. M. Salkovskis, J. W. Kirk, et al). Oxford:Oxford University Press.

- \& Warwick, H. M. (1986) Morbid preoccupations, health anxiety and reassurance: a cognitive behavioural approach to hypochondriasis. Behaviour Research and Therapy, 24, 597-602.

Sanderson, W. C., Beck, A. T. \& McGinn, L. K. (1994) Cognitive therapy for generalised anxiety disorder: significance of co- morbid personality disorders. Journal of Cognitive Psychotherapy: An International Quarterly, 8, 13-18.

Simons, A. D., Lustman, P. J., Wetzel, R. D., et al (1985) Predicting response to cognitive therapy of depression: The role of learned resourcefulness. Cognitive Therapy and Research, 9, 79-89.

Stern, R. \& Marks, I. M. (1973) Brief and prolonged flooding: a comparison in agoraphobic patients. Archives of General Psychiatry, 28, 270-276.

\section{Multiple choice questions}

1 Exposure therapy:

a Is the treatment of choice in obsessivecompulsive disorder

b Is less effective if the patient has severe depression

c Has to be carried out in the presence of the therapist

d Has not been shown to be effective at longterm follow-up

e Is more effective if graded and prolonged

2 Cognitive therapy for depression:

a Is always more effective if combined with antidepressants

b May be less effective with severe depression

c Is not appropriate for poorly educated patients

d Should not be considered if the patient has only mildly negative thinking

e Cannot be applied to in-patients

3 Factors associated with positive outcome in CBT include:

a Learned resourcefulness

b Pre-therapy level of anxiety or depression

c Compliance with self-help assignments

d High intelligence

e Frequent, intrusive negative thoughts

4 The general psychiatrist can help to prepare a patient for CBT by:

a Treating severe anxiety or depression

b Explaining the nature of cognitive/ behavioural treatment

c Giving the patient literature about the therapy

d Looking at the advantages and disadvantages of therapy for a patient who is ambivalent

e Interpreting unconscious resistance

\section{MCQ answers}

$\begin{array}{llllllll}\text { 1 } & & \text { 2 } & & 3 & & 4 & \\ \text { a } & \text { T } & \text { a } & \text { F } & \text { a } & \text { T } & \text { a } & \text { T } \\ \text { b } & \text { T } & \text { b } & \text { T } & \text { b } & \text { T } & \text { b } & \text { T } \\ \text { c } & \text { F } & \text { c } & \text { F } & \text { c } & \text { T } & \text { c } & \text { T } \\ \text { d } & \text { F } & \text { d } & \text { F } & \text { d } & \text { F } & \text { d } & \text { T } \\ \text { e } & \text { T } & \text { e } & \text { F } & \text { e } & \text { F } & \text { e } & \text { F }\end{array}$

